\title{
Finite Element Analysis of Cracked Flexible Pavements Embedded with Controlled Low-Strength Material Bases
}

\author{
Te-Wen Tu, a , Her-Yung Wang ${ }^{2, b}$, Li-Jeng Huang ${ }^{2, c}$ \\ ${ }^{1}$ Department of Mechanical Engineering, Air Force Institute of Technology, Kangshan District 82063, \\ Kaohsiung City, Taiwan, R.O.C. \\ ${ }^{2}$ Department of Civil Engineering, National Kaohsiung University of Applied Sciences, \\ Sanming District 80778, Kaohsiung City, Taiwan, R.O.C.

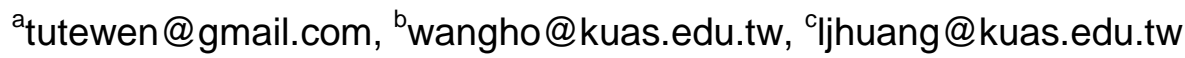

Keywords: CLSM, FEM, Fracture Mechanics, Flexible Pavements

\begin{abstract}
Cracks resulting from shrinkage due to temperature or moisture usually exist in the flexible pavements in highways and runways of airport. This paper presents at finite element analysis of 4-layered flexible pavements with a penetrated longitudinal crack on surface layer embedded with controlled low-strength materials (CLSM) bases which are well-known recycling sustainable materials. Two-dimensional deformation assumption is employed. Emphasis is put on the effect of using CLSM bases on the reduction surface settlement, horizontal and vertical stresses as well as crack face displacements. Two kinds of CLSMs, i.e., CLSM-B80/30\% and CLSM-B130/30\% are compared with graded crushed stones and AC used for base materials. Numerical study shows that vertical settlements, vertical and horizontal stresses by using CLSM-B130/30\% base is smaller than that by using graded crushed stone and is shown to be good material employed as base substitute for graded crushed stone in flexible pavement design.
\end{abstract}

\section{Introduction}

In the past decade, the controlled low-strength materials (CLSM) had been widely applied to rapid construction and repair of flexible pavements in highway engineering [1,2]. CLSM is a kind of flowable fill defined as self-compacting cementitious material that is in a flowable state at the initial period of placement and has a specified compressive strength of 1200 psi or less at 28 days or is defined as excavatable if the compressive strength is 300 psi or less at 28 days [3]. The special features of CLSM include: durable, excavatable, erosion-resistant, self-leveling, rapid curing, flowable around confined spacing, wasting material usage and elimination of compaction labors and equipment, etc. The authors have also conducted some preliminary studies on engineering properties of CLSM [4] and the numerical analyses on static and free vibration analysis of CLSM bases in flexible pavements $[5,6]$.

A crack resulting from shrinkage due to temperature or moisture usually exists in the flexible pavements in highways and runways of airport. The existing surface crack may propagate, grow and increase the occurrence of rutting of the pavement. It is well known that analytical methods, computational schemes and experimental skills based on linear elastic fracture mechanics (LEFM) have been applied for stress analysis near crack tip for homogeneous and isotropic materials [7]. The crack analyses for multi-layered flexible pavements are limited to the numerical analyses [8,9].

This study focused at static analysis of 4-layered flexible pavements with a surface crack via using finite element method. The top layer is considered as asphalt concrete (AC); while 4 kinds of base materials are employed, i.e., graded crushed stones, CLSM-B80/30\%, CLSM-B130/30\% and AC. Comparison study is performed on the vertical displacement, vertical stress and horizontal stress as well as the crack opening and overall principal stress distribution due to the existence of the surface-layer-through crack. Estimation of stress intensity factors (SIF) is also attempted. 


\section{Finite Element Modeling of 4-Layered Flexible Pavements}

Classical displacement-based finite element equations employed for static analysis can be expressed as

$$
[K]\{x\}=\{f\}
$$

where, $\{x\}$ and $\{f\}$ are systematic nodal displacement vector and force vector, respectively; and the stiffness matrix is expressed as

$$
[K]=\int_{V}[B]^{T}[D][B] d V
$$

In which $[B]$ is strain-displacement matrix and $[D]$ is the constitutive matrix containing elastic modulus and Poisson's ratio.

It is well known that there are many approaches for estimating stress intensity factors by using finite element methods: (a) extrapolation of displacements near crack tip; (b) extrapolation of stresses near crack tip; (c) domain integral; (d) contour integral [7-11]. However, approaches (a) and (b) require finer meshes and (a) is better than (b). In the preliminary study of SIFs of flexible pavement, we will employ approaches (a) and (d).

SIF Determination based on Displacement Calculation [7]. For plane strain, stress intensity factor of Mode-I can be obtained from displacement component such as

$$
K_{I}=\frac{G u_{1}}{\sqrt{\frac{r}{2 \pi}} \cos \frac{\theta}{2}\left[1-2 v+\sin ^{2} \frac{\theta}{2}\right]}
$$

SIF Determination using Contour Integral [10, 11]. The stress intensity factor can also be calculated from contour integral method as

$$
K_{I}=\sqrt{2 \pi} A_{1}=\sqrt{2 \pi} \int_{\Gamma}\left(W T_{F E}-u_{F E} T^{W}\right) d S
$$

where, $W$ and $T^{W}$ are defined as follows:

$$
\begin{aligned}
& W=\frac{\rho^{-1 / 2}}{\pi(2 \kappa-1)}\left[\begin{array}{c}
\left(\kappa-\frac{1}{2}\right) \cos \frac{\theta}{2}-\frac{1}{2} \cos \frac{3 \theta}{2} \\
\left(\kappa+\frac{1}{2}\right) \sin \frac{\theta}{2}-\frac{1}{2} \sin \frac{3 \theta}{2}
\end{array}\right], \quad T_{F E}=\left[\begin{array}{c}
\sigma_{X X} \cos \theta+\sigma_{X Y} \sin \theta \\
\sigma_{X Y} \cos \theta+\sigma_{Y Y} \sin \theta
\end{array}\right]_{F E} \\
& T^{W}=\frac{-G \rho^{-3 / 2}}{\pi(2 \kappa-1)}\left[\begin{array}{c}
\left(\frac{3}{2} \cos \frac{\theta}{2}+\frac{1}{2} \cos \frac{5 \theta}{2}\right) \cos \theta+\left(\frac{1}{2} \sin \frac{5 \theta}{2}-\frac{1}{2} \sin \frac{\theta}{2}\right) \sin \theta \\
\left(\frac{1}{2} \sin \frac{5 \theta}{2}-\frac{1}{2} \sin \frac{\theta}{2}\right) \cos \theta+\left(\frac{5}{2} \cos \frac{\theta}{2}-\frac{1}{2} \cos \frac{5 \theta}{2}\right) \sin \theta
\end{array}\right], u_{F E}=\left[\begin{array}{l}
u_{x} \\
u_{y}
\end{array}\right]_{F E}
\end{aligned}
$$

with $\kappa=3-4 v$ for plane strain and $\kappa=(3-v) /(1+v)$ for plane stress.

\section{Numerical Results}

The 4-layered flexible pavement considered is illustrated in Fig. 1(a). Different materials in base layer along with all the parameters in all profiles are listed in Table 1. The material constants for AC, graded crushed stone, compacted soil and natural soil are the same as those employed for typical highway in Taiwan [12]. The material constants for CLSM-B80/30\% and CLSM-B130/30\% are obtained from experimental work as explained in [13]. The vertical concentrated axle load, $Q_{0}=40 \mathrm{kN}(9000 \mathrm{lbs})$ is assumed. This is equivalent to a single axle load for a common AASHTO HS20 truck. 


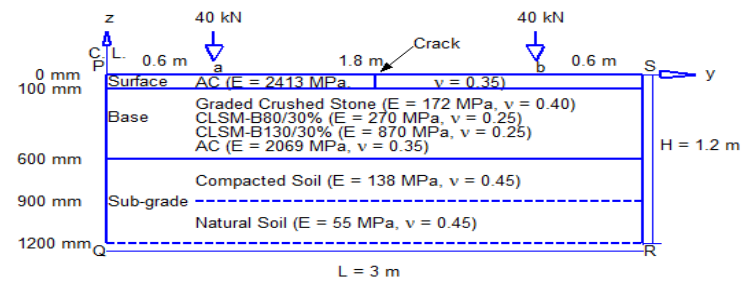

(a) Schematic of 4-layered flexible pavement

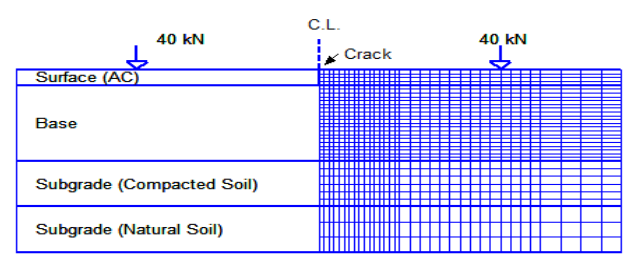

(b) FEM mesh of 4-layered flexible pavement

Fig. 1 Schematic and FE mesh of a 4-layered flexible pavement with a surface crack.

Table 1. Material Properties and Vertical Model Dimensions.

\begin{tabular}{|c|c|c|c|c|}
\hline Layer \# & Materials & Thickness $(\mathrm{mm})$ & $E(M P a)$ & $v$ \\
\hline 1 (Surface) & AC & 100 & 2413 & 0.35 \\
\hline 2 (Base) & Graded Crushed Stone & 500 & 172 & 0.40 \\
& CLSM-B80/30\% & & 270 & 0.25 \\
& CLSM-B130/30\% & & 870 & 0.25 \\
& AC & 300 & 2413 & 0.35 \\
\hline 3 (Sub-grade) & Compacted Soil & 300 & 138 & 0.45 \\
\hline 4(Sub-grade) & Natural Soil & & 55 & 0.45 \\
\hline
\end{tabular}

The $Q 4$ finite elements are employed for the displacement and stress analysis. Due to symmetry only one-half of domain is analyzed and totally $34 \times 33=1122$ rectangular elements along with $35 \times 34=1190$ nodes are employed for numerical calculation by using program coded in MATLAB (Fig. 1(b)). No special elements are employed but only finer meshes used near the cracked region with higher stress gradients.

The calculated overall deformation plots and crack face displacements of flexible pavement with a surface crack by using 4 kinds of different base materials are shown in Fig. 2(a) and 2(b), respectively. The pavement using CLSM-B130/30\% base performs well for settlement resistance and smaller crack opening than graded crushed stone base. The vertical displacements, vertical stresses and horizontal stresses can be observed in Fig. 3(a), 3(b) and 3(c), respectively. The von-Mises iso-stress lines for overall and near crack-tip local domain are shown in Fig. 4(a) and 4(b), respectively. Stress concentration occurs in both crack tip and loading point as expected. The results of flexible pavement using CLSM-B130/30\% base are similar to those using AC base.
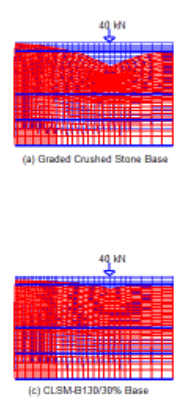
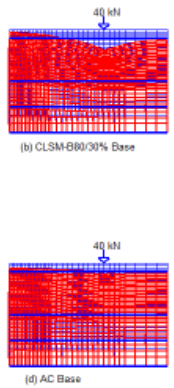

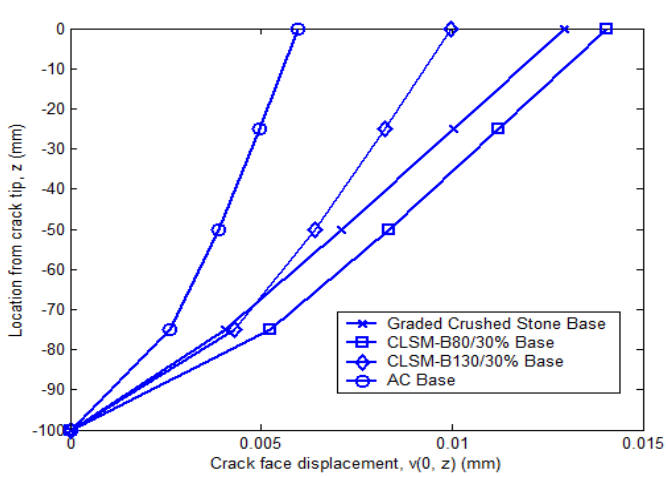

(a) Displacement profiles for difference bases (b) Crack face displacement for difference bases Fig. 2 Calculated displacements and crack openings of flexible pavement with a surface crack. 


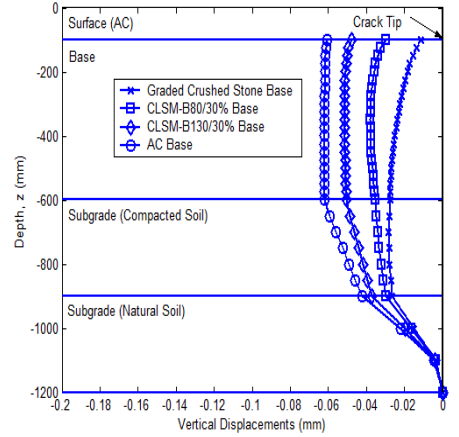

(a) Vertical displacements

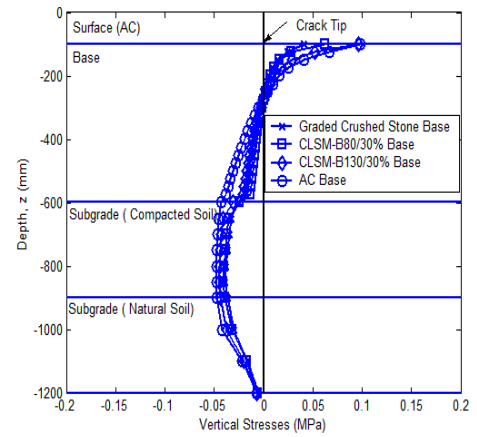

(b) Vertical stresses

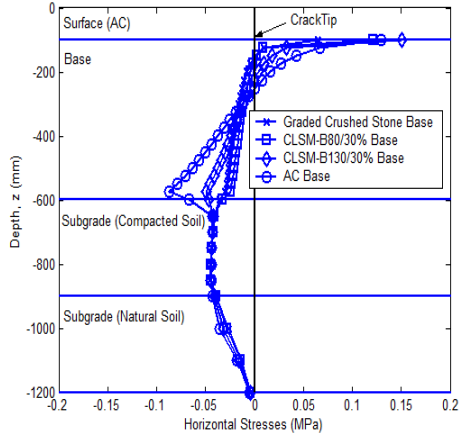

(c) Horizontal stresses

Fig. 3 Displacements and stress in 4-layered flexible pavement with a surface crack.
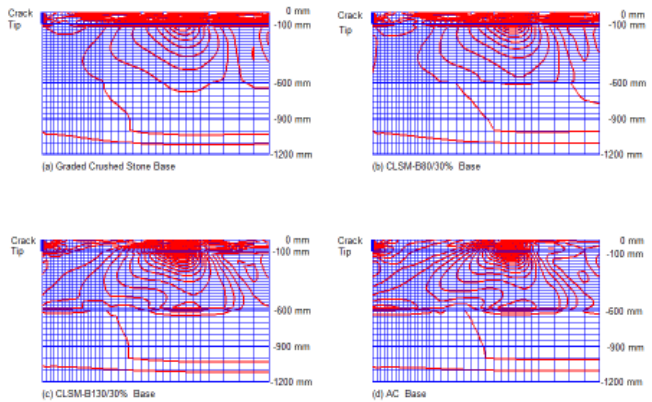

(a) Global iso-stress lines
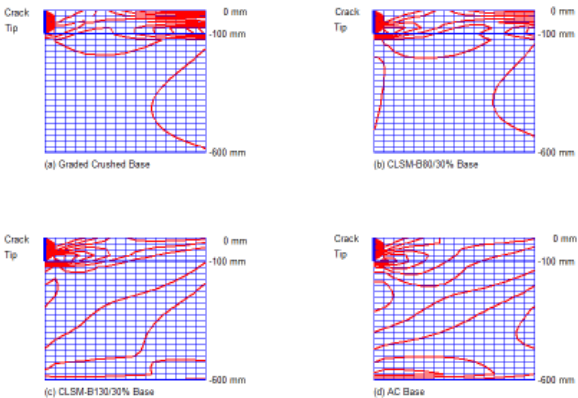

Fig. 4 von-Mises stresses in the 4-layered flexible pavement with a surface crack.

We then attempt to estimate the stress intensity factors by using the FEM with current meshes. The SIF determination based on displacement calculation, Eq. (3), is shown in Fig. 5(a) while the SIF calculated from contour integral, Eq. (4), is depicted in Fig. 5(b). In general, the estimated SIFs are higher for stiffer materials. The calculated SIFs from contour integrals using different paths become constant for pavements using AC surface and AC base as expected. The estimated SIFs obtained from displacement calculation and averaged results from contour integrals for flexible pavement are shown in Table 2. We should emphasize that more and finer meshes are required for calculation of displacement-based SIFs and current results can only be a preliminary result.

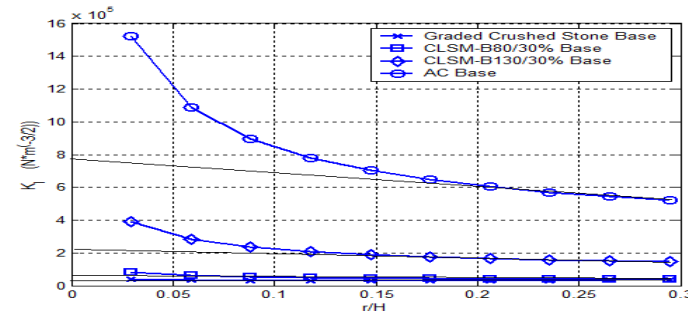

(a) $K_{I}$ calculated from displacements

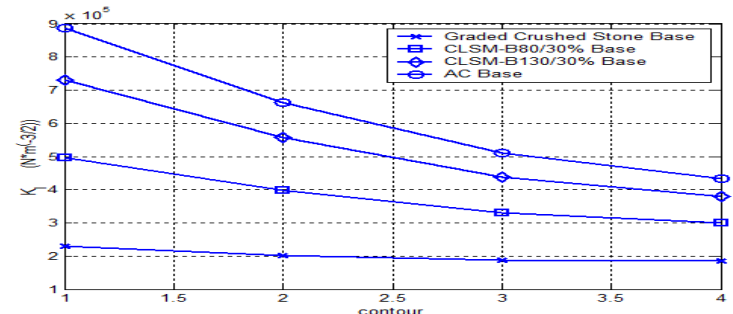

(b) $K_{I}$ calculated from contour integrals

Fig. 5 Calculated stress intensity factors for 4-layered flexible pavement with a surface crack.

Table 2. Calculated stress intensity factors for flexible pavement with a surface crack.

\begin{tabular}{|c|c|c|c|c|}
\hline & $\begin{array}{c}\text { Graded Crushed } \\
\text { Stone Base }\end{array}$ & $\begin{array}{c}\text { CLSM- } \\
\text { B80/30\% Base }\end{array}$ & $\begin{array}{c}\text { CLSM- } \\
\text { B130/30\% Base }\end{array}$ & AC Base \\
\hline From Eq. (3) & 0.4 & 0.7 & 2.2 & 7.6 \\
\hline From Eq. (4) & 2.008 & 3.810 & 5.261 & 6.233 \\
\hline
\end{tabular}




\section{Conclusion}

Comparative study on the displacement and stress within 4-layered flexible pavement embedded with 4 kinds of base materials with an existing surface crack has been conducted using finite element analyses. Some concluding remarks are:

(a) Displacements and stresses for flexible pavements using CLSM bases (B80/30\% and B130/30\%) are bounded between those using graded crushed stone base and AC base;

(b) The crack face opening of cracked pavement using CLSM-B130/30\% base is smaller than that using graded crushed stone base;

(c) CLSM-B130/30\% shows to be a good material employed as the base substitute for graded crushed stone in flexible pavement design considering both the resistance of settlement and cost.

\section{References}

[1] D. F. Lin, H. L. Luo, H. Y. Wang, M. J. Hung, Successful Application of CLSM on a Weak Pavement Base/Subgrade for Heavy Truck Traffic, Perform. Constr. Facil. 21(1) (2007) 70-77.

[2] M. Bassani, S. Khosravifar, D. G. Goulias, C. W. Schwartz, Long-term Resilient and Permanent Deformation Behavior of Controlled Low-Strength Materials for Pavement Applications, Transport. Geotech. 2 (2015) 108-118.

[3] ACI-229R, Controlled Low-Strength Materials (Reproved 2005), Farmington Hills (MI) 2005.

[4] L. J. Huang, Y. N. Sheen, D. H. Le, On the Multiple Linear Regression and Artificial Neural Networks for Strength Prediction of Soil-Based Controlled Low-Strength Material, $3^{\text {rd }}$ International Conference for Advanced Materials Design and Mechanics and Workshop on Android Robotics, Singapore, May 23-24, 2014.

[5] L. J. Huang, H. Y. Wang, W. L. Huang, M. C. Lin, Finite Element Analysis of Controlled Low-Strength Material Bases Part I- Static Analysis, ISEC-9, Resilient Structures and Sustainable Construction, Valencia, Spain, 24th-29th, July, 2017 (Accepted).

[6] L. J. Huang, H. Y. Wang, W. L. Huang, M. C. Lin, Finite Element Analysis of Controlled Low-Strength Material Bases Part II- Free Vibration Analysis, ISEC-9, Resilient Structures and Sustainable Construction, Valencia, Spain, 24th-29th, July, 2017 (Accepted).

[7] G. C. Sih, Mechanics of Fracture, Vol. 1: Methods of Analysis and Solutions of Crack Problems, Noordhoff International Publishing, Leyden, The Netherlands, 1973.

[8] D. Bozkurt, Three-dimensional Finite Element Analysis to Evaluate Reflective Cracking Potential in Asphalt Concrete Overlays, $\mathrm{PhD}$ Dissertation, University of Illinois, Urbana-Champaign, USA, 2003.

[9] F. L. Tsai, R. L. Lytton, S. I. Lee, Prediction of Reflection Cracking in Hot Mix Asphalt Overlays, [C]. Proceedings of 2010 Annual Meeting of the Transportation Research Board (TRB) Conference, Washington, D. C., USA, 2010.

[10] B. Szabo, I. Babuska, Finite Element Analysis, New York, 1991.

[11] C. Castle, An Investigation of the Through Thickness Stress Intensity Factor Using P- and H-Element Finite Element Analysis, Master Thesis, The University of Wisconsin-Milwaukee, 2012.

[12] D. W. Chang, C. E. Chang, Study on Data Interpretation and Structural Index of Flexible Pavement, Tamkang J. Sci. Eng. 1(2) (1998) 83-96.

[13] Y. N. Sheen, L. J. Huang, D. H. Le, Engineering Properties of Controlled Low-Strength Material Made with Residual Soil and Class F Fly Ash, $3^{\text {rd }}$ International Conference for Advanced Materials Design and Mechanics and Workshop on Android Robotics, Singapore, May 23-24, 2014. 